\title{
Modelo social y límites al crecimiento en el antropoceno
}

\author{
Luis Moreno \\ Consejo Superior de Investigaciones Científicas (CSIC-IPP) \\ luis.moreno@csic.es \\ Daniele Conversi \\ Basque Foundation of Science y EHU-UPV \\ daniele.conversi@ehu.es
}

DOI: https://doi.org/10.20318/eunomia.2017.3657

El Antropoceno es una nueva era geológica provocada por la acción de los seres humanos. Pese a sus diversas interpretaciones, hay un creciente consenso en situar a mediados del pasado siglo $\mathrm{XX}$ el comienzo del nuevo período geológico (Zalasiewicz et al., 2015). Fue desde entonces, y no por una coincidencia casual, cuando el consumo de masas, entendido como una pulsión a obtener más de lo necesario, ha conformado el rumbo de las sociedades contemporáneas provocando efectos destructores en el ambiente muy superiores a los anteriores impactos producidos por la industrialización. Existe ahora un acuerdo científico prácticamente unánime de que el calentamiento climático global ha sido inducido linealmente por las pautas desenfrenadas del consumo humano (Cook et al., 2013). Ello es consecuencia de una idea de progreso abocada a la catástrofe socioambiental.

\section{Los límites al crecimiento}

El primer informe auspiciado por el Club de Roma de 1972 puso de manifiesto que existían unas barreras naturales y ambientales a la expansión del industrialismo y del consumo masivo que no deberían ignorarse si quería preservarse el futuro de la vida social en nuestro planeta Desde entones, la prevalente ideología consumista ha redoblado la promoción de un individualismo posesivo que reduce las relaciones ciudadanas en nuestras democracias a un cálculo personal e interesado. Tales prácticas apuntan institucionalmente al aniquilamiento del modelo social sustentado en el Estado del Bienestar (EB), auténtico hito civilizatorio del siglo XX en Europa.

Ya a fines del siglo XIX el sociólogo estadounidense Thorstein Veblen (18571929 ) identificó las pautas del consumismo ostensible (conspicuous consumerism) (Veblen, 1899). Los análisis de Veblen se realizaron en una época en los que la posibilidad de imitar la vida de los ricos era muy limitada, pero la situación cambió en la segunda mitad del siglo XX. Fue a partir de entonces que el tipo de consumismo se intensificó conllevando un gasto "compulsivo" de dinero a fin de adquirir bienes y servicios en procesos de emulación pecuniaria y capacidad de 
renta, los cuales establecían los mecanismos de obtención y preservación del estatus social de las personas.

La última ideología neoliberal consumista de vocación global concierne a patrones homogéneos por lo que el consumismo no debería ser considerado solo como una fenómeno individualizado resultado de las elecciones voluntarias de las personas, sino como resultado contextual de un proceso inducido de alcance mundial (Sklair, 2012). El consumismo a escala global se ha articulado como un proceso unidireccional de homogeneización cultural, de resultas del cual nunca antes tantos individuos habían participado en los hábitos de consumo de las viejas elites occidentales (Conversi, 2012). Así, y tras decenios de post-fordismo se incrementaron exponencialmente el consumo de energía generada por combustibles fósiles y la utilización masiva, por ejemplo, de automóviles altamente contaminantes de la atmósfera.

Tras el tránsito al tercer mileno, y corolario del descontrol de los mecanismos de crédito privado y la maximización de prácticas especulativas mediante sofisticados instrumentos financieros y derivados, se desencadenó en 2007 una crisis global provocada por el colapso de las hipotecas subprime en el mercado de la vivienda estadounidense. La subsiguiente licuación financiera provocó una crisis mundial de amplio alcance y repercusiones que afectó, especialmente, a las democracias del bienestar (post-) industriales, cuyos gobiernos pusieron a disposición del sistema bancario cantidades ingentes de dinero público a fin de evitar la quiebra de sus sistemas financieros. El alcance y efectos futuros del crack del 2007 son de naturaleza conjeturable, pero la extensión mundial del tipo de "capitalismo de casino" ha constatado una estrategia hacia un tipo de crecimiento donde la avidez imposibilita la solidaridad característica del welfare.

Tales tendencias han agudizado los procesos de exclusión social, no sólo en los países menos desarrollados y más empobrecidos. Según Oxfam-Intermón la gran mayoría de las víctimas y perdedores del cambio climático son precisamente aquellas que viven en países que contribuyen en menor medida al cambio climático (Oxfam, 2015). Considérese que el $10 \%$ del los hogares más ricos del mundo emiten alrededor de 24 toneladas de CO2, porcentaje que se compara con el $50 \%$ de los hogares más pobres. Así mismo, el $1 \%$ de los más hogares estadounidenses, singapurenses, luxemburgueses o saudíes con rentas más altas están entre los mayores emisores individuales con más de 200 toneladas. Es inadecuada, por tanto, una visión simplista de fractura entre Este y Oeste, o Norte y Sur ya que en el $1 \%$ mencionado hay que incluir también a las élites súper ricas de China, Rusia, India o Brasil, pongamos por caso. Esta nueva geografía del cambio climático, de desigualdad de rentas y de exclusión social hace necesaria, por tanto, una acción concertada de todos los países (Piketty y Chancel, 2015).

\section{El modelo europeo bajo asedio}

El Modelo Social Europeo (MSE) se estructuró con la institucionalización del Estado del Bienestar (EB) desarrollado en el Viejo Continente durante el siglo XX. Está articulado en torno a los valores de equidad social (igualdad), solidaridad colectiva (redistribución) y eficiencia productiva (optimización). Tales valores promueven la ciudadanía social entendida como aspiración a una vida digna y al bienestar social de los individuos mediante la provisión social en situaciones de riesgo. EI MSE auspicia el crecimiento económico sostenible basado en la cohesión social. En el orden global, el MSE está asediado por otros sistemas de desarrollo económico y social alternativos, como son el "neoesclavismo" emergente asiático, el cual propone el dumping social como valor añadido de crecimiento económico, y la 
remercantilización individualista anglo-norteamericana, que auspicia la compra del bienestar por los propios ciudadanos.

La versión contemporánea del "neoesclavismo" implica el control de las personas con el propósito de su explotación económica (Bales, 1999). El sistema extendido en el continente asiático no se refiere strictu sensu a la propiedad física de las personas, sino a la capacidad de controlarlas como artefactos para generar beneficios materiales, lo que comporta una exacerbación de la desigualdad. Piénsese, por ejemplo, que en 2009 apenas 50 oligarcas con fortunas superiores al millardo de dólares estadounidenses controlaban en la India el equivalente al $20 \%$ de su PIB y el $80 \%$ de su capitalización bursátil. Tales datos contrastaban con la lucha por la supervivencia de más de 800 millones de sus compatriotas, los cuales disponían de menos de un dólar al día.

El "neoesclavismo" cabe ser entendido como una sinécdoque de las relaciones de poder entre ciudadanos pudientes, detentadores de los medios de apropiación de la riqueza y del poder político, y los ciudadanos precarios, vendedores de su fuerza de trabajo para su pervivencia existencial. En pos de la maximización de sus inversiones, algunas corporaciones multinacionales eligen aquellas localizaciones dispuestas a facilitar su asentamiento, bien sea productivo o financiero, con la oferta de un menor nivel impositivo, de una liberalización de las condiciones de trabajo y de ausencia de restricciones a los impactos negativos en el medio. El resultado suele materializarse en unas prácticas de deslocalización y de dumping social entendido como una limitación de las condiciones laborales y de los derechos sociales de los trabajadores --cuando no de su eliminación-- para poder competir en mejor condiciones de mercado con los países europeos, obligando a éstos a adoptar medidas de competencia a la baja (race to the bottom) inasumibles a no ser que procedan a desmantelar sus sistemas de bienestar y ciudadanía social.

Por su parte, el sistema de remercantilización individualista, característico de los países anglo-norteamericanos proclama la liberación de las personas de sus ligámenes colectivos, emplazándoles a la construcción autónoma de sus propias biografías vitales. En el límite de tal perspectiva, los ciudadanos pierden su sentido de la lealtad institucional y rehúyen la solidaridad con sus prójimos más allá de las mecánicas rutinarias y los hábitos colectivos, lo que aumenta su asociabilidad. Para un proyecto vital asocial no se necesitan mayores compromisos ciudadanos y los individuos gestionan los recursos relacionales atendiendo a su único provecho, y utilizándolos discrecionalmente à la carte. Paradójicamente productos y bienes culturales puestos a la venta y alcance de su libre decisión individual devienen objetos homogéneos y normalizados colectivamente, lo que incentiva el voraz consumismo estandarizado (Moreno, 2012).

Ambos modelos "neoesclavista" y remercantilizador apuntan a la aceleración de los efectos perversos del cambio climático. No es casual que, respectivamente, EEUU y China e India hayan sido países refractarios a poner límites al proceso de calentamiento global. Sin embargo, en diciembre de 2015 se firmó finalmente el Acuerdo de París que entró en vigor en noviembre de 2016 (Klein et al., 2017). El documento recoge una amplia gama de recomendaciones de políticas públicas y requiere de los países firmantes que revisen periódicamente sus niveles y actualicen sus acciones al respecto. La Unión Europea tomó el liderazgo en las negociaciones que superaron momentos críticos de desacuerdo entre algunos de los 174 países participantes. La conversaciones celebradas en Marrakech en 2016 han proseguido la monitorización del Acuerdo de París y no sólo a la financiación general, sino muy especialmente respecto al apoyo prometido a los países en desarrollo que más 
dificultades encuentras para romper la relación perversa entre desigualdad y exclusión social.

\section{Enfoques interdisciplinares}

Se requiere un cambio de paradigma acorde con el dramático tránsito del Holoceno al Antropoceno. Las ciencias sociales se han rezagado en la elaboración de estudios y propuestas, tanto teóricas como prácticas para afrontar el cambio climático. En los años 1990, cuando aún era posible actuar con carácter preventivo a fin de evitar males mayores, solo una minoría de investigadores sociales fue capaz de identificar las consecuencias de unos efectos tan potencialmente nocivos para el bienestar de los seres humanos. Los acontecimientos se han acelerado y la situación general se ha deteriorado. Solo ahora se denota un mayor interés en una convergencia interdisciplinar, la cual se ha plasmado en la aparición de nuevos campos analíticos como la economía ecológica (ecological economics), la gestión de la sostenibilidad (management of sustainability) o la producción más limpia (cleaner production). Con algunas excepciones recientes, todavía están ausentes los trabajos interdisciplinares de estudiosos de las ciencias humanas y sociales que estimulen el debate y ofrezcan alternativas en conjunción con las ciencias llamadas "duras".

En lo que afecta al área del welfare o bienestar social, se hace imperativa la asunción de una visión más amplia y generalizable que incluya los riesgos ambientales junto a los tradicionales riegos sociales a los que el MSE afrontó con éxito con la institucionalización del EB (analfabetismo, enfermedad o jubilación, pongamos por caso). La preservación de un modelo social que respete un desarrollo sostenible y la defensa de la ciudadanía social activa son cruciales para el bienestar social. Consiguientemente, la adecuación del cambio climático a las necesidades humanas también implica erradicar la pobreza, el hambre y la desnutrición. La necesidad de desarrollar un modelo económico sostenible conlleva, en suma, el desarrollo de un modelo del bienestar legitimado en una sociedad justa e inclusiva.

La democracia deliberativa europea requiere de un mayor entendimiento de las opciones en juego (Ugarriza y Caluwaerts, 2014). El masaje mediático (McLuhan y Fiore, 1967) es tan poderoso que la lucha de las ideas se presenta en modo desequilibrado, dado el poder de los intereses de las grandes corporaciones y conglomerados financieros, principales inductores de los nocivos efectos del cambio climático. Pero como ha sucedido en el pasado, nuevas posibilidades y formas comunicativas favorecen la contraposición de otras ideas al sacralizado "pensamiento único". Además, en un mundo crecientemente interconectado, las ideas y acciones propagadas telemáticamente posibilitan un contrapunto de ideas, intereses e instituciones altamente efectivo. La conjunción de ambas dimensiones virtual y real, o cosmopolita y local, constituye un elemento crucial de movilización para el cambio y permanencia del modelo de bienestar en Europa. Es también responsabilidad de los investigadores de las ciencias sociales y humanas proveer de enfoques superadores de la inevitabilidad de la catástrofe socioambiental.

\section{Bibliografía}

BALES, K. (1999): Disposable People. New Slavery in the Global Economy, University of California Press, Berkeley (CA).

CONVERSI, D. (2012): "Modernism and nationalism", Journal of Political Ideologies, $17(1), 13-34$. 
COOK, J. (2013): "Quantifying the consensus on anthropogenic global warming in the scientific literature", Environmental Research Letters, 8 (2), 1-7 (024024).

KLEIN, D., et al. (2017): The Paris climate agreement : analysis and commentary, Oxford University Press, Oxford.

McLUHAN, M. y FIORE, Q. (1967): The medium is the massage. An inventory of effects. Nueva York: Bantam Books [Ed. castellano: El medio es el masaje. Un inventario de efectos. Buenos Aires: Paidós, 1969]

MORENO, L. (2012): La Europa Asocial. Crisis y Estado del Bienestar, en nsula, Barcelona.

OXFAM (2015): Extreme Carbon Inequality, Oxfam, Oxford. En línea: https://www.oxfam.org/en/research/extreme-carbon-inequality.

PIKETTY, T. y CHANCEL, L. (2015): Carbon and inequality: from Kyoto to Paris. Trends in the global inequality of carbon emissions (1998-2013) \& prospects for an equitable adaptation fund, Paris School of Economics, París.

SKLAIR, L. (2012): “Culture-Ideology of Consumerism”, en G. Ritzer (ed.) The WileyBlackwell Encyclopedia of Globalization, John Wiley y Sons, Hoboken (NJ).

UGARRIZA, J. E. y CALUWAERTS, D. (eds.) (2014): Democratic Deliberation in Deeply Divided Societies: From Conflict to Common Ground, Palgrave Macmillan, Houndmills, Basingstoke.

VEBLEN, T.(1899): The Theory of the Leisure Class, Macmillan, Londres [Ed. Castellano (1944), Teoría de la clase ociosa, Fondo de Cultura Económica, México DF].

ZALASIEWICZ, J. et al. (2015): "When did the Anthropocene begin? A mid-twentieth century boundary level is stratigraphically optimal", Quaternary International, 383: 196-203. 